\title{
Resection of a cardiac hemangioma in a patient with chest pain
}

\author{
Naritomo Nishioka ${ }^{1}$, Ryushi Maruyama ${ }^{1}$, Kosuke Ujihira $^{1}$, Yutaka I ba $^{1}$, Eiichiro Hatta ${ }^{1}$, Akira \\ Yamada $^{1}$, Yoshihiko Kurimoto ${ }^{1}$, Hiroko Noguchi ${ }^{2}$, Toshiya Shinohara ${ }^{2}$, Katsuhiko Nakanishi ${ }^{1}$ \\ 1. Department of Cardiovascular Surgery, Teine Keijinkai Hospital, Hokkaido, Japan. 2. Department of Pathology, Teine \\ Keijinkai Hospital, Hokkaido, Japan.
}

Correspondence: Naritomo Nishioka, MD. Address: Department of Cardiovascular Surgery, Teine Keijinkai Hospital, 12-1-40, Maeda 1-jou, Teine-ku, Sapporo, Hokkaido, Japan. E-mail: naritomo.n@nifty.com

Received: J une 16, 2015

DOI : $10.5430 /$ crcp.v2n4p80

Accepted: September 25, 2015

URL: http://dx.doi.org/10.5430/crcp.v2n4p80

\section{Abstract}

This is a case of a 77-year-old woman who presented complaining of chest pain and was found to be secondary to a mass in her left atrial appendage (LAA). The patient underwent complete surgical excision of the $4.5 \mathrm{~cm}$ mass and the pathology report revealed a cardiac hemangioma. Her postoperative clinical course was uneventful and she was discharged in good condition and chest-pain-free.

\section{Key words}

Cardiac hemangioma, Left atrial appendage, Chest pain

\section{I ntroduction}

Cardiac hemangiomas are rare, benign, and primitive tumors that occur with an incidence of $0.8 \%-1.6 \%$ among all detected benign heart tumors. They arise from the cardiac ventricles, atria, or, rarely, the valves or epicardium ${ }^{[1]}$. The clinical presentation (palpitations, dyspnea, arrhythmia, or sudden death) varies according to the tumor's location and size ${ }^{[2]}$. Most cardiac masses (including hemangiomas), however, are asymptomatic and are discovered incidentally by imaging, such as transthoracic or transesophageal echocardiography (TTE or TEE), computed tomography (CT), or cardiac magnetic resonance imaging (MRI). This case report describes the resection of a cardiac hemangioma in the left atrial appendage (LAA) in a woman presenting with chest pain.

\section{Case report}

A 77-year-old woman with no significant past medical history presented with a one-month's history of anginal chest pain in the setting of exertional dyspnea. Physical examination revealed stable vital signs and normal heart sounds without murmurs. Her electrocardiogram showed a normal sinus rhythm with non-specific ST and T wave changes. A TTE identified a large left atrial (LA) mass consistent with a possible intra-cardiac myxoma. A CT confirmed a $4.5 \mathrm{~cm}$ mass within the LAA (see Figure 1). The coronary angiography revealed a large tortuous abnormal feeder vessel that supplied the LA tumor and arose from the circumflex branch of the left coronary artery. 
Figure 1. Computed tomography with an axial view showed the LAA tumor's measuring $4.5 \mathrm{~cm} \times 3.0 \mathrm{~cm}$ with an enhanced left atrial side (arrow). The feeding artery was not clear.

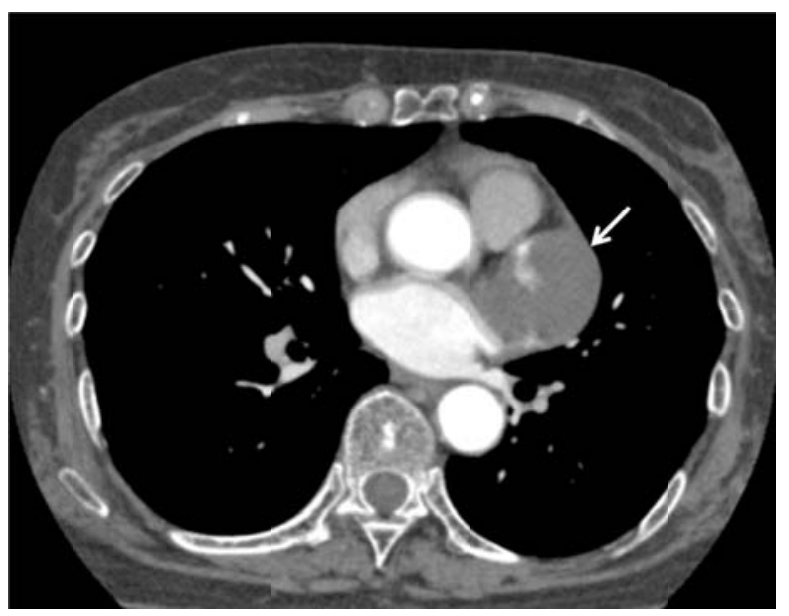

Figure 2. Intra-surgical macroscopic view of tumor

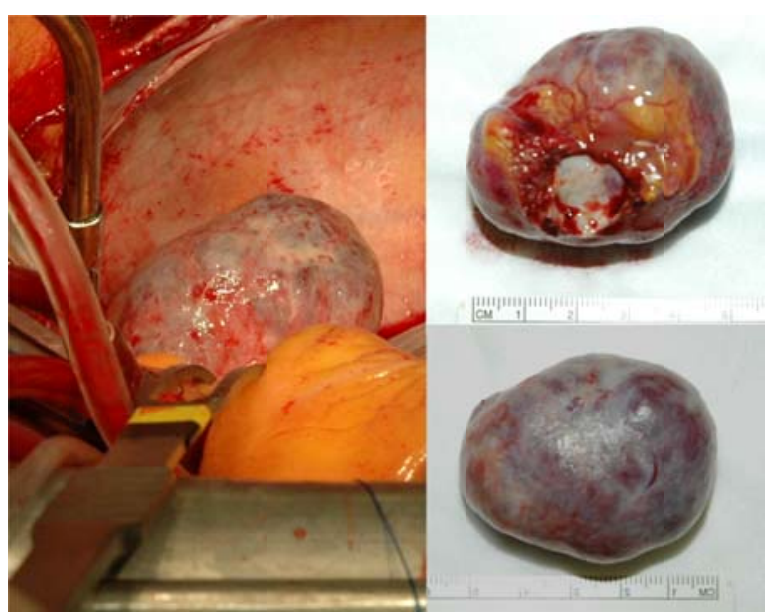

Figure 3. Histological exam showed a hemangioma with a mixture of cavernous and capillary vessel types (Hematoxylin \& eosin; $\times 100$ )

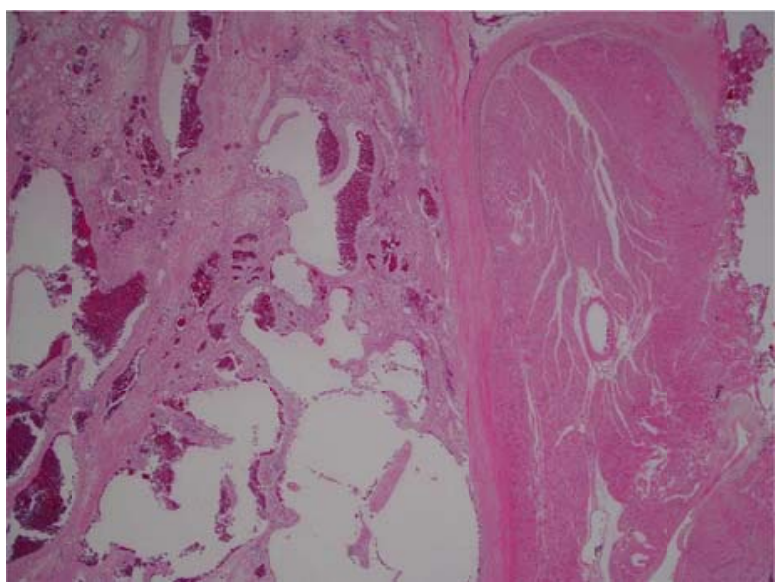

The patient underwent resection of the LAA tumor. A median sternotomy was made, and the parietal pericardium was normal. During the conventional cardiopulmonary bypass (CPB), the aortic cannula was inserted and bicaval venous cannulation was performed. The CPB was established, and the vent cannula was placed via the right upper pulmonary vein. The ascending aorta was clamped while the cold blood cardioplegia was infused through the aortic root and coronary sinus; cardiac arrest and myocardial protection were thus obtained. No tissue adhesion was seen surrounding the tumor, and mobilization of the tumor was possible without any complications. A trans-septal approach was used to examine the LA. Furthermore, because the tumor $(4.5 \mathrm{~cm} \times 3.0 \mathrm{~cm})$ only involved the LAA and the LA was intact, the whole tumor was easily resected with the excision of the LAA (see Figure 2). Pathological results revealed a cardiac hemangioma with a 
mixture of cavernous and capillary types without malignancy (see Figure 3). The postoperative course was uneventful, and the patient was discharged without chest pain on postoperative day 15.

\section{Discussion}

Cardiac hemangiomas occur rarely and account for $5 \%-10 \%$ of benign primary cardiac tumors and $2 \%$ of all cardiac tumors ${ }^{[3]}$. Symptoms result from embolism or compression of the surrounding structures. The most common presentation is exertional dyspnea; however, arrhythmias, pseudoangina, and signs of right heart failure can also occur. Some cardiac hemangiomas are asymptomatic and are discovered during heart surgery or autopsy ${ }^{[4,5]}$. A large tumor with abundant vascularity, as the LAA hemangioma in the above case, may also present with exertional dyspnea and anginal chest pain without significant coronary artery disease. A probable explanation of such pain is the "coronary artery phenomenon" owing to blood being drawn by the vessel feeding the tumor and away from the major coronary artery.

Diagnoses of cardiac tumors are possible by advanced imaging such as TTE, TEE, CT, and MRI; however, the tumor type diagnosis is seldom possible without histological examination. Cardiac hemangiomas have a non-specific pattern on unenhanced CT but in most cases, they show intense enhancement with contrast ${ }^{[6]}$. Cardiac MRI provides a more specific image and may provide a qualitative diagnosis ${ }^{[7]}$. CT is helpful for surgical planning because it allows submillimeter spatial resolution and identifies mass effect on adjacent structures. Most importantly, CT can demonstrate whether pericardial wall is involved or not.

In our case, the cardiac tumor was seen in echocardiography when the patient presented for exertional dyspnea and chest pain. Preoperatively, the cardiac tumor was evaluated with a CT scan and coronary angiography. The CT demonstrated a partially enhanced mass and the coronary angiography showed a tumor blush, both of which are typical findings; however, even with these data, the final nature of the tumor could not be diagnosed.

Cardiac hemangiomas may grow in the pericardium, endocardium, or myocardium. In our patient, the pathology revealed that the mass probably originated from the myocardium because of a coating within the myocardium without continuation into the pericardium or epicardium.

Complete resection is the treatment of choice for cardiac hemangiomas, and the prognosis is generally favorable with a low rate of recurrence. There is long term survival reported even with an incomplete resection of the tumor ${ }^{[1]}$. In a previous case report, the right anterolateral mini-thoracotomy approach with minimally invasive cardiac surgery (MICS) was used to resect a cardiac hemangioma ${ }^{[8]}$. We decided against the MICS approach in our patient because of the location of the tumor. Instead, the cardiac surgery was performed with a typical median sternotomy and cardiopulmonary bypass and we were successful in resecting the tumor in its entirety. The histological exam revealed cardiac hemangioma. The postoperative course was uneventful and the patient remained asymptomatic.

\section{Conclusion}

Cardiac hemangiomas are rare, benign, and primitive tumors of the heart. The histological diagnosis cannot be predicted by imaging, however, in these patients, contrast-enhanced CT and coronary angiography are still necessary for a correct morphological characterization needed to guide the surgical approach. Complete surgical resection, when possible, is the best approach to treat a cardiac hemangioma, and the postoperative outcome is generally favorable.

\section{References}

[1] Botha J, Ihlberg L, Eithenawy A, et al. A giant cavernous hemangioma of the heart. Ann Thorac Surg. 2010; 90: 293-5. PMid: 20609803. http://dx.doi.org/10.1016/j.athoracsur.2009.10.077 
[2] Patel J, Sheppard MN. Sudden death owing to right atrial hemangioma. J Forensic Sci. 2011; 56: 529-30. PMid: 21265841. http://dx.doi.org/10.1111/j.1556-4029.2010.01667.x

[3] Abraham KP, Reddy V. Neoplasms metastatic to the heart: review of 3314 consecutive autopsies. Am J Cardiovasc Pathol. 1990; 3: 195-8. PMid: 2095826.

[4] Brizard C, Latremouille C, Jabara VA, et al. Cardiac hemangiomas. Ann Thorac Surg. 1993; 56: 390-4. http://dx.doi.org/10.1016/0003-4975(93)91193-Q

[5] Asako T, Masahiro S, Takuya F. Resection of a giant cardiac hemangioma. Gen Thorac Cardiovasc Surg. 2013; 61:353-5. PMid: 23104457. http://dx.doi.org/10.1007/s11748-012-0145-9

[6] Grebenc ML, Rosando de Christenson ML, Bruke AP, et al. Primary cardiac and pericardial neoplasms: radiologicpathologic correlation. Radiographics. 2000; 20: 1073-103. PMid: 10903697. http://dx.doi.org/10.1148/radiographics.20.4.g00j1081073

[7] Jude E, Philip SL, Paul AV, et al. Pericardial hemangioma taking origin from the posterior wall of the left atrium. Ann Thorac Surg. 2009; 87: 54-6. PMid: 19463571. http://dx.doi.org/10.1016/j.athoracsur.2009.03.005

[8] Elisabetta L, Michele DB, Lucia T, et al. Cavernous hemangioma of the tricuspid valve: minimally invasive surgical resection. Ann Thorac Surg. 2003; 76: 2097-9. http://dx.doi.org/10.1016/S0003-4975(03)00742-2 